\title{
Technology-based interventions in health care
}

\author{
J. M. Kane ${ }^{1,2}$ \\ ${ }^{1}$ Psychiatry Research, The Zucker Hillside Hospital, North Shore - Long Island Jewish Health System, 75-59 263rd Street, Glen Oaks, NY, USA \\ ${ }^{2}$ Hofstra North Shore LIJ School of Medicine, Hempstead, NY, USA
}

\begin{abstract}
There are several converging forces that create a particularly opportune time for technological solutions to enhance cost efficiency in healthcare. Health care costs are unsustainable, yet many patients do not have adequate access to state-ofthe-art treatments or to ongoing disease management. Consumerism is an increasingly powerful force in healthcare and the emphasis on personalised medicine will help to define future research and clinical treatment strategies. At the same time, the phenomenal advances in internet utilisation and mobile device applications provide possibilities that have never before existed. We have reason to be very optimistic about these opportunities, but appropriate research will be required to develop scalable and sustainable methods as well as determine expected outcomes.
\end{abstract}

Received 20 April 2014; Revised 21 May 2014; Accepted 22 May 2014; First published online 22 August 2014

Key words: eHealth, health technology, mHealth, mobile devices.

\section{Introduction}

There is enormous need to increase access, reduce disparities and create greater cost efficiencies in healthcare. This presents particular challenges in the area of mental illness, where inadequate public knowledge, lack of insight, stigma and inconsistent reimbursement, etc. add additional obstacles.

Estimates suggest that one out of every four people will experience a mental illness and that a substantial proportion never receive treatment. Among those who are diagnosed and treated, there are often inordinate and costly (from a disease and societal perspective) delays, not just for weeks or months, but in many cases years (Compton et al. 2007). In addition, many chronic and severe mental illnesses (such as schizophrenia and bipolar disorder) have their onset in late adolescence or early adulthood adding another array of challenges for early detection and intervention (Kessler et al. 2007). When examining the leading causes of disability or loss of quality adjusted life years, mental illnesses and addictive disorders account for an enormous share (Murray \& Lopez, 1996). This is a consequence of not only their prevalence and severity, but also the inconsistencies in access to quality care.

It has also become increasingly clear that a disproportionate share of medical expenditures are driven by a relatively small proportion of patients with

Address for correspondence: John M. Kane, MD, Psychiatry Research, The Zucker Hillside Hospital, North Shore - Long Island Jewish Health System, 75-59 263rd Street, Glen Oaks, NY, USA.

(Email: psychiatry@nshs.edu) co-morbid medical and psychiatric conditions as well as psychosocial and environmental obstacles to consistent and coordinated care. Increased emphasis on a true integration of primary medical and psychiatric care has been a missed opportunity for many years, but it is possible that the Affordable Care Act which became law on 23 March 2010 will provide better and broader coverage to those affected and create enhanced incentives for optimum integration.

It is in this context that we need to consider the role of technology in improving the efficiency and effectiveness of systems of care (Steinhubl et al. 2013). For the purposes of this discussion, we will consider technologies which assist: the patient (and family or caregiver); the treating clinicians; the hospital, clinic (or other healthcare setting); and the payer. In addition, we can divide the domains of interest into: screening and access to services; treatment delivery and monitoring; patient self-assessment and disease management; and quality assurance/performance improvement.

\section{High-throughput screening}

The availability of web-based screening tablet or smart phone apps, which can allow large numbers of people to review their own subjective wellbeing and illness symptoms in a user friendly and non-intrusive fashion is an enormous potential assest in the struggle for more timely identification of mental illness (BenZeev, 2012). Web-based surveys can be applied in schools and universities, by large employers and other organisations interested in increasing the early identification and treatment of those with psychiatric 
and addictive disorders (Loewy et al. 2012). The use of self-administered screening tools with mobile devices or a computer kiosk when making a visit to a primary care physician or specialist can help to screen large numbers of individuals without imposing on precious time with health professionals. Thresholds can be established for brief interventions and referral. The screening, brief intervention and referral to treatment (SBIRT) model which has been widely used in substance abuse is an example (Fleming et al. 2002).

Such surveys can also provide links to psychoeducational web sites and other resources. The availability of accurate, evidenced based information on the web is another challenge (Birnbaum et al. in press). Prospective patients can be vulnerable to all types of misinformation and screening tools need to be linked to appropriate resources. The use of such tools also allows for epidemiologic surveys which can be conducted in real time and provide estimates of prevalence, severity and other metrics. These data can of course be helpful in public health planning, resource allocation, etc. as well as monitoring the success in linking people in need to appropriate resources.

There are initiatives underway to screen social media communications in order to identify bullying or various types of psychopathology (New York Times, 2013). This raises a number of ethical issues, but much of social media is public and, therefore, this is a natural outgrowth of such phenomena.

\section{Treatment delivery and monitoring}

There are widespread shortages of well-trained mental health personnel, particularly in areas such as child and adolescent or geriatric psychiatry. The use of telemedicine can offer considerable advantages in terms of increasing access and efficient use of practitioners' time. Even with those patients who are experiencing a psychotic episode, live, two-way video has been shown to be acceptable and reliable, with little or no decrement in the clinician's ability to accurately assess the nature and severity of relevant signs and symptoms (Zarate et al. 1997; Shen et al. 2008). From the perspective of the health care system, reducing the need for $24 \mathrm{~h}$ a day, 7 days a week, in-person coverage of emergency rooms in many community hospitals can reduce expenses substantially. Utilising two-way video in those circumstances where leaving the home is an impediment to seeking and receiving services, e.g., post-partum depression, agoraphobia, etc. can also help to reach those people who would otherwise be difficult to engage or retain in treatment. In addition, it has been suggested that a substantial proportion of office visits do not require face-to-face interaction and could be done via two-way video, particularly in psychiatry. This could reduce the costs and inconvenience associated with travel, facilitate off-hour visits which might be more convenient for many who have difficulty taking time off from work, and, thereby, reduce the rate of no-shows and cancelled appointments. Office visits can also be reduced when large numbers of patients can be tracked by mobile devices with abnormalities or significant changes in their conditions relayed to their clinicians with the potential for text messages, e-mails, telephone calls or links to instructional web sites reducing the potential need for an office or clinic visit.

The lack of consistent availability of practitioners to deliver specific types of therapy, such as cognitive behaviour therapy or family and patient psychoeducation for patients suffering from schizophrenia (Gottlieb et al. 2013), can be addressed by providing web-based tools for patients to access and utilise at their own convenience, thereby reducing need for practitioner time as well as increasing overall access (Rotondi et al. 2010). Though concerns have been raised about diminishing the clinician patient relationship, such options serve to extend clinicians' reach and empower patients to do as much as possible on their own with necessary backup from professionals. In addition, it can improve access to evidence-based techniques that vary in availability depending on geography, etc.

There is certainly considerable demand on the part of consumers for health and wellness resources, which suggests the acceptability of such technological approaches; however, there is also an important concern as suggested previously that such resources need to be designed and/or vetted by responsible professionals or oversight bodies. As suggested by Steinhubl et al. (2013), there are tens of thousands of healthcare related apps available for download, whereas the food and drug administration (FDA) has reviewed approximately 100 . This raises the concern that apps might be made available to patients which are not necessarily helpful and might even be harmful (Wolf et al. 2013). It is incumbent upon academic medicine and professional societies as well as state and federal agencies responsible for overseeing healthcare to be proactive in assuring that technological advances live up to their potential, while at the same time reducing risks associated with irresponsible and non-evidence-based applications.

\section{Patient self-assessment and disease management}

If patients can facilitate their own diagnoses and monitor their own illnesses as well as access and benefit from treatments that lend themselves to selfadministration, the implications for cost efficiency and personalisation are enormous. It is very difficult 
for clinicians to have an accurate picture of the severity and frequency of various subjective experiences. Experience sampling or ecological assessment methods can be used on a broad scale to create real time data that are useful in establishing and confirming diagnosis as well as monitoring response to treatment and the natural history of untreated illness. Hence mHealth (mobile health) technologies can enhance consumer convenience and satisfaction while at the same time empowering patients to participate in the management of their own condition and facilitating better control over chronic illnesses (Swendsen et al. 2011). For clinics and health care systems as well as payers, the ability to reduce the risk of relapse or exacerbations and the resultant increase in the use of expensive emergency services or inpatient hospitalisation is of enormous potential importance. Monitoring patients in real time (passively or actively) with mobile devices can provide early signs to caregivers or health professionals, which can be used to trigger an intervention that might prevent or at least delay the need for increasingly costly levels of care (Spaniel et al. 2008). This provides personalised monitoring well beyond the intermittent visits to a clinic or office.

Numerous studies across healthcare and especially in the management of psychiatric disorders have identified the actual taking of medication as prescribed as one of the major challenges in promoting public health. Non-adherence plays a tremendous role in precipitating avoidable visits to emergency departments and unnecessary hospital admissions. Osterberg \& Blaschke (2005) in their comprehensive review of this topic, suggested that, of all medication-related hospital admissions in the USA, 33-69\% are due to poor medication adherence, with a resulting cost of approximately $\$ 100$ billion per year. The inability of either patients or physicians to accurately estimate the degree of adherence is limited. Existing methods that are the simplest (questioning the patient or having them complete a self-assessment form) tend to be inaccurate, whereas those that are potentially more accurate (blood levels, urine markers, etc.) tend to be intrusive, costly and not easily scalable. The new digital health technologies such as the 'digital health feedback system' (Kane et al. 2013) offers tremendous advantages. In this approach, a digital tablet consisting of the patient's medication with an ingestion sensor that is embedded in each tablet and activated by stomach fluids after ingestion allows for a unique signal to be communicated from the sensor to an adhesive receiver worn on the torso which automatically logs the date and time of each ingestion. The wearable sensor can also collect data on activity levels and physiologic measures such as heart rate. Such a system can provide valuable information to carers and clinicians, but of equal importance can provide the patient with data to help empower and inform their own efforts at disease management.

\section{Quality assurance and performance improvement}

As healthcare reform places more and more emphasis on linking quality of care, patient satisfaction and better outcomes to actual payments for services, the need to monitor these domains in a seemless, meaningful, consistent and inexpensive fashion is of greater importance than ever. The capabilities that new technologies bring to this effort are enormous. Patients' symptoms, wellbeing and overall level of functioning can be tracked in real time with appropriate data capture and analysis providing needed metrics to all components of the healthcare system. Patient (and family/caregiver) satisfaction can be obtained with greater ease than ever before. The ability to aggregate and analyse large data sets to inform service development and delivery, resource allocation, physician (and other health professional) continuing education, patient and family education can add enormously to the overall success and cost-effectiveness of the health care enterprise.

\section{Summary and conclusions}

Clearly, we are in the midst of one of the great transformations in health care. The use of new technologies has the potential to alter every component of the health care enterprise while enhancing quality, fostering patient convenience and empowerment and at the same time reducing overall costs.

The ways in which we take advantage of this opportunity, including the thoroughness and thoughtfulness of our ongoing research and evaluation will have a profound effect on the lives of present and future generations.

\section{References}

Ben-Zeev D (2012). Mobile technologies in the study, assessment, and treatment of schizophrenia. Schizophrenia Bulletin 38, 384-385.

Birnbaum ML, Candan K, Pascucci O, Libby I, Kane JM (in press). The impact of online resources and social media on help seeking behavior in youth with psychotic symptoms. Early Intervention in Psychiatry.

Compton MT, Carter T, Bergner E, Franz L, Stewart T, Trotman H, McGlashan TH, McGorry PD (2007). Defining, operationalizing, and measuring the duration of untreated psychosis: advances, limitations and future directions. Early Intervention in Psychiatry 1, 236-250.

Fleming MF, Mundt MP, French MT, Manwell LB, Stauffacher EA, Barry KL (2002). Brief physician advice for problem drinkers: long-term efficacy and benefit-cost analysis. Alcohol Clinical Experimental Research 26, 36-43. 
Gottlieb JD, Romeo KH, Penn DL, Mueser KT, Chiko BP (2013). Web-based cognitive-behavioral therapy for auditory hallucinations in persons with psychosis: a pilot study. Schizophrenia Research 145, 82-87.

Kane JM, Perlis RH, DiCarlo LA, Au-Yeung K, Duong J, Petrides G (2013). First experience with a wireless system incorporating physiologic assessments and direct confirmation of digital tablet ingestions in ambulatory patients with schizophrenia and bipolar disorder. Journal of Clinical Psychiatry 74, e533-e540.

Kessler RC, Angermeyer M, Anthony JC, DE Graaf R, Demyttenaere K, Gasquet I, DE Girolamo G, Gluzman S, Gureje O, Haro JM, Kawakami N, Karam A, Levinson D, Medina Mora ME, Oakley Browne MA, Posada-Villa J, Stein DJ, Adley Tsang CH, Aguilar-Gaxiola S, Alonso J, Lee S, Heeringa S, Pennell BE, Berglund P, Gruber MJ, Petukhova M, Chatterji S, Ustün TB (2007). Lifetime prevalence and age-of-onset distributions of mental disorders in the World Health Organization's World Mental Health Survey Initiative. World Psychiatry 6, 168-176.

Loewy RL, Therman S, Manninen M, Huttunen MO, Cannon TD (2012). Prodromal psychosis screening in adolescent psychiatry clinics. Early Intervention in Psychiatry 6, 69-75.

Murray CJ, Lopez AD (1996). The Global Burden of Disease. WHO, World Bank, Harvard School of Public Health, Harvard University Press: Boston, MA.

New York Times (2013). Warily, Schools watch students' on the internet. 28 October 2013. Retrieved 20 May 2014 from http://www.nytimes.com/2013/10/29/technology/ some-schools-extend-surveillance-of-students-beyondcampus.html?_r=0.
Osterberg L, Blaschke T (2005). Adherence to medication. New England Journal of Medicine 353, 487-497.

Rotondi AJ, Anderson CM, Haas GL, Eack SM, Spring MB, Ganguli R, Newhill C, Rosenstock J (2010). Web-based psychoeducational intervention for persons with schizophrenia and their supporters: one-year outcomes. Psychiatric Services 61, 1099-1105.

Shen J, Kobak KA, Zhao Y, Alexander MM, Kane JM (2008). Use of remote centralized raters via live 2-way video in a multicenter clinical trial for schizophrenia. Journal of Clinical Psychopharmacology 28, 691-693.

Spaniel F, Vohlídka P, Hrdlicka J, Kozený J, Novák T, Motlová L, Cermák J, Bednarík J, Novák D, Höschl C (2008). ITAREPS: information technology aided relapse prevention programme in schizophrenia. Schizophrenia Research 98, 312-317.

Steinhubl SR, Muse ED, Topol EJ (2013). Can mobile health technologies transform health care? Journal of the American Medical Association 310, 2395-2396.

Swendsen J, Ben-Zeev D, Granholm E (2011). Real-time electronic ambulatory monitoring of substance use and symptom expression in schizophrenia. American Journal of Psychiatry 168, 202-209.

Wolf JA, Moreau JF, Akilov O, Patton T, English III JC, Ho J, Ferris LK (2013) Diagnostic inaccuracy of smartphone applications for melanoma detection. JAMA Dermatology 149, 422-426.

Zarate Jr CA, Weinstock L, Cukor P, Morabito C, Leahy L, Burns C, Baer L (1997). Applicability of telemedicine for assessing patients with schizophrenia: acceptance and reliability. Journal of Clinical Psychiatry 58, 22-25. 